\title{
Die Bedeutsamkeit individueller Merkmale für die Entwicklung von Rechtschreibkompetenzen in der Grundschule
}

\author{
Jessica Jaeuthe, Jenny Lenkeit, Stefanie Bosse, Katja Bogda und Nadine Spörer \\ Humanwissenschaftliche Fakultät, Institut für Lehrerbildung, Universität Potsdam
}

\begin{abstract}
Zusammenfassung: Aus theoretischer Sicht existieren verschiedene Merkmale, die einen Einfluss auf die individuelle Entwicklung der Rechtschreibkompetenz von Grundschulkindern ausüben können. Um diese zu analysieren, wurden in der vorliegenden Studie Schreibungen von 607 Schülerinnen und Schülern zu drei Messzeitpunkten (Anfang Klasse 2, Ende Klasse 2, Ende Klasse 3) untersucht. Zur Bestimmung des Rechtschreibkompetenzniveaus wurden Schülerinnen und Schüler mittels latenter Profilanalyse zu den drei Profilen Buchstabenentdeckende (überwiegend nicht lautgetreue Schreibungen), Phonemprofis (überwiegend lautgetreue Schreibungen) und Rechtschreibspezialisten (vor allem korrekte Schreibungen) zugeordnet. Mittels logistischer Regressionen wurde geprüft, welche Zusammenhänge zwischen den individuellen Merkmalen Geschlecht, kognitive Grundfähigkeiten und Arbeitsverhalten und den Zuordnungen zu einem Profil sowie den Wechseln zwischen den Profilen bestehen. Unter Kontrolle des Arbeitsverhaltens zeigten sich keine signifikanten Zusammenhänge mit dem Geschlecht oder der kognitiven Grundfähigkeit. Hingegen konnten bedeutsame Zusammenhänge des Arbeitsverhaltens sowohl mit der Zuordnung zu den Profilen als auch mit den Wechseln zwischen den Profilen Phonemprofis und Rechtschreibspezialisten gefunden werden.

Schlüsselwörter: Rechtschreibkompetenz, Längsschnittstudie, Logistische Regression, Kompetenzniveau, Arbeitsverhalten
\end{abstract}

The Relevance of Individual Characteristics on the Development of Spelling Competence of Primary School Children

Abstract: From a theoretical point of view, there are various characteristics influencing the individual development of spelling competence of primary school children. In the present study, 609 students were examined at three measurement points (beginning of class 2, end of class 2 , end of class 3 ). To determine the spelling competence level, latent profile analyses were used to assign the students to three profiles 1) predominantly phonemically incorrect spelling, 2) predominantly phonemically correct spelling and 3) predominantly orthographically correct spelling. Logistic regression analyses included gender, basic cognitive skills, and working behavior as predictors for the assignment to one of the competence levels as well as the change between levels across time. Controlling for working behavior no significant effects of gender or basic cognitive skills are found. However, students' working behavior significantly influences not only the assignment to the higher levels of spelling competency but also changes between predominantly phonemically correct and predominantly orthographically correct across time.

Keywords: spelling competence, longitudinal study, logistic regression, level of competence, working behavior

Rechtschreibung wird gesellschaftlich eine bedeutende Rolle für zukünftigen Bildungserfolg zugeschrieben. Zugleich gelingt es nicht allen Schülerinnen und Schülern bis zum Ende der Schulzeit fehlerfrei schreiben zu können (z.B. Valtin, Badel, Löffler, Meyer-Schepers \& Voss, 2003). Schülerinnen und Schüler entwickeln sich erwartungsgemäß unterschiedlich schnell. Worauf diese unterschiedliche Entwicklung zurückzuführen ist, wurde jedoch bislang nicht hinreichend erforscht. Theoretisch sind verschiedene Merkmale denkbar, die einen Einfluss auf die Entwicklung der Rechtschreibkompetenz nehmen können. So konnte in zahlreichen Studien ermittelt wer- den, dass spezifische Einflussfaktoren, wie phonologische Bewusstheit, einen positiven Einfluss auf die Entwicklung schriftsprachlicher Leistungen zeigten (Pfost, 2015). Darüber hinaus konnten positive Einflüsse der kognitiven Grundfähigkeiten von Schülerinnen und Schülern auf die Rechtschreibkompetenzentwicklung nachgewiesen werden (z. B. Dummert, Endlich, Schneider \& Schwenck, 2014; Zöller \& Roos, 2009). Inwiefern weitere unspezifische Merkmale von Bedeutung sein könnten, wurde hingegen bisher kaum untersucht. Es liegen erste Hinweise dafür vor, dass positive Zusammenhänge zwischen dem Arbeitsverhalten und der Rechtschreibleistung bestehen 
(z. B. Kikas \& Silinskas, 2016). Jedoch ist bislang unklar, inwiefern diese Zusammenhänge auch für die Entwicklung entlang der Rechtschreibkompetenzniveaus gelten, wobei insgesamt ein Mangel an Studien besteht, welche nicht nur die Entwicklung der Rechtschreibleistung der Schülerinnen und Schüler messen, sondern auch die Kompetenzniveaus der Rechtschreibkompetenzentwicklung berücksichtigen. In der vorliegenden Arbeit soll überprüft werden, inwiefern die Entwicklung der Rechtschreibkompetenz vom Geschlecht, der kognitiven Grundfähigkeit sowie vom Arbeitsverhalten der Schülerinnen und Schüler abhängig ist.

\section{Theoretischer und empirischer Hintergrund}

\section{Rechtschreibkompetenz}

Rechtschreibkompetenz setzt sich aus verschiedenen Aspekten zusammen (KMK, 2005). Dazu gehört, dass Schülerinnen und Schüler selbst Fehler finden und diese korrigieren, aber insbesondere die Fähigkeit, Rechtschreibstrategien anzuwenden (KMK, 2005). Zu den Rechtschreibstrategien gehören laut der KMK (2005) die Fähigkeit, Wörter lautgetreu zu schreiben und später auch orthographische und morphematische Regelungen zu berücksichtigen.

Es existieren verschiedene theoretische Modelle, die beschreiben, wie sich die Rechtschreibkompetenz von Schülerinnen und Schülern entwickeln soll (z.B. Brügelmann \& Brinkmann, 1994; Scheerer-Neumann, 1989; Thomé, 2003; für eine Übersicht siehe Jaeuthe et al. 2020; Siekmann \& Thomé, 2012). Auch wenn in diesen Modellen unterschiedliche Begriffe verwendet werden, so herrscht Konsens darüber, dass es sich bei der Rechtschreibkompetenz um hierarchisch aufeinander aufbauende Kompetenzen handelt. Darüber hinaus werden in allen Modellen folgende Kompetenzniveaus benannt:

1. Es entstehen erste Schreibprodukte, wobei die Schreibung noch nicht der Lautung des Wortes entspricht.

2. Es entstehen Schreibungen, die der Lautung des Wortes entsprechen (lautgetreue Schreibungen).

3. Es entstehen orthographisch korrekte Schreibungen.

Diese Abfolge betrifft das Schreiben unbekannter Wörter. Es ist durchaus möglich, dass Kinder schon Wörter aus dem Gedächtnis korrekt schreiben. Versuchen sie jedoch ein neues Wort zu schreiben, so ist dies in der Regel noch nicht lautgetreu. Erst durch die Auseinanderset- zung mit Rechtschreibung entwickeln sich lautgetreue und anschließend orthographisch korrekte Schreibungen.

Die Entwicklung von Rechtschreibkompetenz ist ein langwieriger Prozess, bei dem korrekte Schreibungen erst am Schluss stehen. Soll untersucht werden, auf welchem Stand der Entwicklung sich die Schülerinnen und Schüler befinden, so genügt es nicht, die Anzahl der richtig geschriebenen Wörter zu betrachten. Vielmehr muss der gesamte Prozess der Rechtschreibkompetenzentwicklung mit den drei zuvor genannten Niveaustufen berücksichtigt werden.

\section{Einflussfaktoren auf Rechtschreibkompetenz}

Die schulische Leistung von Schülerinnen und Schülern ist von verschiedenen Faktoren abhängig. Nach Baumert, Blum und Neubrand (2004) wirken diese Faktoren auf der Ebene der Gemeinschaft und des Schultyps, der Schule, der Klasse sowie der Schülerinnen und Schüler bzw. der Lehrperson. Die Rechtschreibkompetenz bildet eine Facette der schulischen Leistung ab. Die vorliegende Arbeit konzentriert sich zur Untersuchung der Rechtschreibkompetenz auf die Ebene der Schülerinnen und Schüler, sodass im Folgenden auf mögliche individuelle Merkmale genauer eingegangen werden soll.

Eine der am häufigsten betrachteten Merkmale im Hinblick auf die Rechtschreibkompetenz ist das Geschlecht. In bisherigen Studien zur Rechtschreibkompetenz konnte vielfach gezeigt werden, dass Mädchen über bessere Leistungen verfügen als Jungen (z. B. Dummert et al, 2014; Schneider, Stefanek \& Dotzler, 1997; Stranghöner et al., 2017; Zöller \& Roos, 2009; bzgl. der Strategien sind Mädchen der Studien zufolge ebenfalls überlegen, z. B. Valtin et al., 2003). Worauf diese Unterschiede zurückzuführen sind, ist bislang nicht eindeutig beantwortet. Möglicherweise liegen die Unterschiede zwischen Mädchen und Jungen in anderen individuellen Merkmalen begründet. Diese können mit Bezug auf die Rechtschreibkompetenz in eher spezifische Merkmale, wie z. B. phonologische Bewusstheit, und eher unspezifische Merkmale, wie z.B. Konzentrationsfähigkeit, kognitive Fähigkeiten und Leistungsmotivation unterteilt werden (Marx, 2007). Der Einfluss spezifischer Einflussfaktoren kann dabei insbesondere für den Beginn der Grundschule als gesichert gelten. So konnten vielfach positive Zusammenhänge und Einflüsse der phonologischen Bewusstheit auf die Entwicklung der Rechtschreibkompetenz gezeigt werden (s. Meta-Analyse von Pfost, 2015 bzw. Einzelstudien von Ennemoser, Marx, Weber \& Schneider, 2012; von Goldammer, Mähler, Bockmann \& Hasselhorn, 2010). Hingegen wurden in bisherigen Untersuchungen 
unspezifische Einflussfaktoren deutlich seltener berücksichtigt, sodass auf diese im Folgenden der Fokus gelegt werden soll.

DiPerna (2006) definiert die unspezifischen Einflussfaktoren als academic enablers und versteht darunter Einstellungen und Verhaltensweisen, welche dazu beitragen, dass sich die Schülerinnen und Schüler stärker in der Schule beteiligen und somit mehr vom Unterricht und den Instruktionen profitieren. Dabei werden folgende vier Lernermöglicher unterschieden: interpersonale Merkmale (teilen, helfen), Motivation (intrinsisch, Zielorientierung und Selbstwirksamkeitserwartung), Engagement (im Sinne von Mitarbeit) und akademische Fähigkeiten (z. B. kognitive Fähigkeiten, die helfen, Informationen möglichst schnell zu erfassen). In der vorliegenden Studie wird insbesondere die Bedeutsamkeit der beiden zuletzt benannten Merkmale berücksichtigt.

\section{Kognitive Grundfähigkeiten}

Kognitive Fähigkeiten können einen entscheidenden Einfluss auf die Entwicklung schulischer Leistungen ausüben. In Anlehnung an die Intelligenztheorie von Cattell und Horn (1978) entspricht die kognitive Grundfähigkeit dem flüssigen Intelligenzfaktor und umfasst die Wahrnehmung komplexer Beziehungen in unbekannten Situationen. Cattell und Horn (1978) grenzen diese von dem kristallinen Intelligenzfaktor $\mathrm{ab}$, welcher das erworbene Wissen, z. B. in der Schule, beinhaltet.

Zusammenhänge zwischen der kognitiven Grundfähigkeit und der Rechtschreibleistung wurden durch zahlreiche Studien belegt. So konnten z.B. Zöller und Roos (2009) für Schülerinnen und Schüler der ersten bis vierten Jahrgangsstufe mittlere positive Korrelationen zwischen der kognitiven Grundfähigkeit und der Rechtschreibleistung zeigen. Ein vergleichbares Zusammenhangsmuster fanden Dummert et al. (2014), die Schülerinnen und Schüler der zweiten bis vierten Jahrgangsstufe in Regelklassen untersuchten. Hingegen zeigten sich bei der längsschnittlichen Untersuchung von Stranghöner et al. (2017) mit 410 Grundschulkindern mit dem Förderschwerpunkt Lernen keine bedeutsamen Zusammenhänge zwischen der kognitiven Grundfähigkeit und der Rechtschreibleistung. Möglicherweise ist dies auf die verringerte Varianz der Ausprägungen beider Konstrukte in der Stichprobe der Schülerinnen und Schüler mit Förderbedarf zurückzuführen.

Bulut (2018) untersuchte längsschnittlich den Einfluss der kognitiven Grundfähigkeit auf die Entwicklung der Rechtschreibkompetenz von 685 Schülerinnen und Schülern der ersten und zweiten Klasse, wobei die unterschiedlichen Strategien mit Hilfe von vier Graphemen (Lupenstellen, z. B. Konsonantenschreibung in $<$ Mund $>$ ) bei der Schreibung von drei Wörtern zu jedem Messzeit- punkt berücksichtigt wurden. Sie konnte zeigen, dass eine höhere kognitive Grundfähigkeit der Schülerinnen und Schüler mit früherer Bewältigung unterschiedlicher Rechtschreibphänomene (phonologisches Prinzip, Auslautverhärtung, Schärfung) zusammenhing. In ihrer Studie klärte die kognitive Grundfähigkeit insgesamt 4.1\% der Varianz in der Rechtschreibentwicklung auf (Bulut, 2018). Es stellt sich jedoch die Frage, inwiefern die Verwendung von drei Wörtern ausreichend ist, um die Rechtschreibkompetenz der Schülerinnen und Schüler zuverlässig einschätzen zu können. Hinsichtlich der Messung der Rechtschreibkompetenz ist zu erwarten, dass die Schülerinnen und Schüler einige Wörter besonders gut kennen, wenn sie diese verinnerlicht oder geübt haben. Um jedoch die Rechtschreibkompetenzniveaus zu messen, ist es notwendig, dass die Schülerinnen und Schüler die Wörter konstruieren, statt sie aus dem Gedächtnis zu schreiben. Insofern ist eine größere Anzahl an Wörtern notwendig, um die unterschiedlichen Kompetenzniveaus valide erfassen zu können.

\section{Arbeitsverhalten}

U. Petermann und F. Petermann (2006) unterscheiden vier Facetten des Arbeitsverhaltens: a) Anstrengungsbereitschaft und Ausdauer, b) Konzentration, c) Selbstständigkeit und d) Sorgfalt beim Lernen. Schülerinnen und Schüler, die über eine hohe Anstrengungsbereitschaft und Ausdauer verfügen, werden z. B. selbst Aufgaben über einen längeren Zeitraum bearbeiten, die für sie wenig interessant sind (U. Petermann und F. Petermann, 2006). Eine hohe Konzentration zeigt sich z.B. durch gezielte Aufmerksamkeit (U. Petermann und F. Petermann, 2006). Unter Selbstständigkeit verstehen U. Petermann und F. Petermann (2006), dass die Schülerinnen und Schüler die Aufgaben allein ausführen, planvoll vorgehen und sich erreichbare Ziele setzen (U. Petermann und F. Petermann, 2006). Sorgfalt bezieht sich auf einen ordentlichen Umgang mit Arbeitsmaterialien und Vollständigkeit bei der Bearbeitung von Hausaufgaben (U. Petermann und F. Petermann, 2006).

Kaufmann (2007) untersuchte die selbsteingeschätzte Lernmotivation und die Anstrengungsmotivation sowie die Rechtschreibleistung von 507 Schülerinnen und Schülern am Ende der dritten und vierten Klasse. Im Ergebnis zeigten sich schwache positive Zusammenhänge zwischen der Anstrengungsmotivation und der Rechtschreibkompetenz, d.h. Schülerinnen und Schüler erzielten bessere Rechtschreibleistungen, wenn sie ihre Arbeitsmotivation positiver einschätzten. Darüber hinaus fanden sich signifikante Unterschiede zwischen Mädchen und Jungen hinsichtlich der Rechtschreibleistung und der Anstrengungsmotivation zu Gunsten der Mädchen, wobei diese Merkmale getrennt voneinander berücksichtigt 
wurden, sodass unklar blieb, inwiefern die Geschlechterunterschiede im Rechtschreiben auf Unterschiede in der Anstrengungsmotivation zurückzuführen waren.

Kikas und Silinskas (2016) verwendeten die Einschätzung von Lehrpersonen zur Ausdauer von 870 Schülerinnen und Schülern am Anfang und Ende der ersten Klasse sowie am Ende der zweiten Klasse. Sie konnten zeigen, dass die Ausdauer mit der Rechtschreibkompetenz hoch korrelierte. Darüber hinaus zeigten Kreuzkorrelationsanalysen, dass nicht nur die Ausdauer die späteren Rechtschreibkompetenzen, sondern auch die Rechtschreibkompetenzen die spätere Ausdauer positiv beeinflussten. Auch in der Studie von Georgiou, Manolitsis, Nurmi und Parrila (2010) erwies sich das durch Lehrpersonen eingeschätzte Arbeitsverhalten (task-focused behavior) in dem Sinne als relevanter Prädiktor der Rechtschreibkompetenz, als dass ein besseres Arbeitsverhalten der Schülerinnen und Schüler mit besseren Rechtschreibkompetenzen einherging. Hierbei muss jedoch berücksichtigt werden, dass diese Studien nicht im deutschsprachigen Raum durchgeführt wurden, sodass Unterschiede auf Grund der verschiedenen Orthographien möglich sind.

Valtin et al. (2003) konnten im Rahmen von IGLU-E ( $N=2951$ Schülerinnen und Schüler) basierend auf Elterneinschätzungen Vorteile des Arbeitsverhaltens zu Gunsten der Mädchen feststellen. Außerdem zeigten in einem Extremgruppenvergleich gute Rechtschreiberinnen und Rechtschreiber ein positiveres Arbeitsverhalten als schwächere Rechtschreiberinnen und Rechtschreiber. Es fehlen jedoch Analysen, in denen sowohl Geschlecht als auch Arbeitsverhalten als simultane Prädiktoren einbezogen wurden, um zu prüfen, ob beide Merkmale eigenständige Einflüsse auf die Rechtschreibkompetenz der Schülerinnen und Schüler aufweisen.

\section{Die vorliegende Untersuchung}

In bisherigen Studien wurde häufig die Anzahl der richtig geschriebenen Wörter berücksichtigt, um die Rechtschreibleistungen $\mathrm{zu}$ bestimmen. Um jedoch die Entwicklung entlang der postulierten Kompetenzniveaus der Rechtschreibung zu untersuchen, ist es notwendig, zwischen nicht lautgetreuen, lautgetreuen und orthographisch korrekten Schreibungen zu unterscheiden. Erst diese qualitative Auswertung ermöglicht einen Nachvollzug der Entwicklung und eine Vorhersage der Wechsel von einem Kompetenzniveau auf das nächsthöhere Kompetenzniveau.

Aus dem empirischen Hintergrund wurde deutlich, dass Geschlechterunterschiede zu Gunsten der Mädchen beste- hen und dass dies auch auf unterschiedliche Kompetenzniveaus zutreffen soll. Daher sollten Mädchen früher als Jungen höheren Kompetenzniveaus zugeordnet werden bzw. früher zu höheren Kompetenzniveaus wechseln.

H1 (Geschlechtsunterschiedshypothese): Mädchen haben höhere Wahrscheinlichkeiten, höheren Kompetenzen zugeordnet zu werden.

Unklar ist, wodurch diese Unterschiede verursacht werden. Bei Betrachtung der eher unspezifischen Einflussfaktoren konnten Vorteile für Schülerinnen und Schüler mit höherer kognitiver Grundfähigkeit und besserem Arbeitsverhalten gezeigt werden. Es stellt sich die Frage, inwiefern diese Merkmale mit der Zuordnung der Schülerinnen und Schüler zu einem Niveau bzw. mit der Entwicklung entlang der Kompetenzniveaus zusammenhängen und inwiefern Geschlechterunterschiede auf Unterschiede bezüglich des Arbeitsverhaltens zurückgeführt werden können. Hinsichtlich der Zusammenhänge der Rechtschreibkompetenzniveaus mit dem Arbeitsverhalten stellt sich jedoch die Frage, ob alle Facetten des Arbeitsverhaltens gleich bedeutsam sind. Insbesondere bei Kompetenzen, die über einen längeren Zeitraum angeeignet werden, wie die Rechtschreibkompetenz, wird vermutet, dass die Schülerinnen und Schüler eine hohe Ausdauer und Anstrengungsbereitschaft benötigen. Dies trifft vor allem dann zu, wenn Graphem-Phonem-Korrespondenzen nicht eindeutig sind. Hingegen könnte eine gute Konzentration für das Erlernen der Laut-BuchstabeZuordnung hilfreich sein (Marx, 2007). Hinsichtlich der deutschen Orthographie wird dahingehend erwartet, dass insbesondere die Ausdauer und Anstrengungsbereitschaft sowie die Konzentration eine Rolle spielen. Hingegen sollten die Aspekte Selbstständigkeit und Sorgfalt für die Entwicklung der Rechtschreibkompetenz weniger bedeutsam sein, sodass diese Facetten in der vorliegenden Studie nicht berücksichtigt werden.

H2 (Statushypothese kognitive Grundfähigkeiten): Schülerinnen und Schüler mit hoher kognitiver Grundfähigkeit haben eine höhere Wahrscheinlichkeit, höheren Kompetenzniveaus zugeordnet zu werden.

H3 (Statushypothese Arbeitsverhalten): Schülerinnen und Schüler mit positivem Arbeitsverhalten haben eine höhere Wahrscheinlichkeit, höheren Kompetenzniveaus zugeordnet zu werden.

H4 (Entwicklungshypothese kognitive Grundfähigkeiten): Schülerinnen und Schüler mit hoher kognitiver Grundfähigkeit haben eine höhere Wahrscheinlichkeit, von einem niedrigen zu einem höheren Kompetenzniveau zu wechseln.

H5 (Entwicklungshypothese Arbeitsverhalten): Schülerinnen und Schüler mit positivem Arbeitsverhalten haben eine höhere Wahrscheinlichkeit, von einem niedrigen $\mathrm{zu}$ einem höheren Kompetenzniveau zu wechseln. 
Die vorliegende Studie steht im Kontext einer größeren längsschnittlichen Untersuchung, in der schulnahe Prädiktoren erfasst wurden, um die Entwicklung schulischer Leistungen nachvollziehen zu können. Der Fokus der vorliegenden Studie liegt dabei auf einem im schulischen Kontext veränderbaren Merkmal - dem Arbeitsverhalten, wobei die Merkmale Geschlecht und kognitive Grundfähigkeit zur Kontrolle mit erhoben wurden. In diesem Projekt bestand nicht die Möglichkeit, spezifische Einflussmerkmale, wie die phonologische Bewusstheit, zu berücksichtigen, sodass in den Analysen für diese nicht kontrolliert werden kann.

\section{Methode}

\section{Stichprobe und Design}

Für die vorliegende Studie konnte auf Daten aus dem längsschnittlichen Evaluationsprojekt „Inklusive Grundschule“ (Spörer et al. 2015) zurückgegriffen werden. Das Projekt hatte das Ziel, individuelle und kontextuale Bedingungen herauszustellen, die einen positiven Einfluss auf die Kompetenzentwicklung von Schülerinnen und Schülern haben können. Für die vorliegende Studie wurden längsschnittliche Testungen aus 30 zufällig gezogenen Grundschulklassen aus dem Bundesland Brandenburg sowie einmalige Befragungen der Lehrpersonen verwendet. Die Messung der Rechtschreibkompetenz der Schülerinnen und Schüler erfolgte zu drei Messzeitpunkten (Anfang zweite Klasse ( $t 1$ ), Ende der zweiten Klasse $(t 2)$ und Ende der dritten Klasse ( $t 3)$ ).

Insgesamt lagen Daten von 697 Schülerinnen und Schülern vor. In der vorliegenden Studie wurden jedoch nur die Schülerinnen und Schüler berücksichtigt, deren Arbeitsverhalten vollständig eingeschätzt wurde. Dadurch reduzierte sich die Stichprobe auf 607 Schülerinnen und Schüler $(t 1=581, t 2=561, t 3=517)$, wobei 482 Schülerinnen und Schüler zu allen drei Messzeitpunkten an der Untersuchung teilnahmen. Die Einschätzung des Arbeitsverhaltens fehlte dabei vor allem von jenen Schülerinnen und Schülern, welche zu $t 1$ noch nicht in den jeweiligen Klassen lernten, sondern aufgrund von familiären Umzügen oder Schulwechseln erst im späteren Verlauf der Untersuchung in die Klassen aufgenommen wurden. Zudem fehlten die Werte einer vollständigen Klasse, da eine Lehrperson keine Angaben zum Arbeitsverhalten ihrer Schülerinnen und Schüler machte. Darüber hinaus verließen Schülerinnen und Schüler frühzeitig die Stichprobe (von $t 1$ zu $t 2: n=42$ bzw. von $t 2$ zu t3: $n=64)$. Hierbei zeigte sich ein systematischer Dropout dahingehend, dass vor allem Schülerinnen und Schüler mit niedrigeren kognitiven Fähigkeiten $(t=$
$2.61, p<.05)$, sowie negativerem Arbeitsverhalten $(t=2.03$, $p<.05)$ die Stichprobe verließen.

$\mathrm{Zu} t 1$ waren die Schülerinnen und Schüler durchschnittlich 7.7 Jahre alt $(S D=0.46)$. Mädchen und Jungen waren gleich häufig vertreten (50\% Mädchen). Fast alle Schülerinnen und Schüler wurden in Deutschland geboren. Lediglich 1\% der Schülerinnen und Schüler gaben ein anderes Herkunftsland an, was für das Bundesland Brandenburg erwartungskonform war. Zusätzlich liegen Angaben der Eltern zum sozio-ökonomischen Hintergrund vor. Hierfür wurden aus den angegebenen Berufstätigkeiten der höchste ISEI der Familie gebildet. Dieser liegt durchschnittlich bei 53.57 ( $S D=18.80$; erstes Quartil 1635, letztes Quartil 67-89), sodass in der Stichprobe eine typische Streuung des sozio-ökonomischen Hintergrundes vorliegt.

\section{Instrumente und Ablauf}

Rechtschreibkompetenz: Die Erfassung der Rechtschreibkompetenz erfolgte zu jedem der drei Messzeitpunkte im Klassenverband und nahm ca. 30 Minuten in Anspruch. Hierfür sollten Schülerinnen und Schüler einzelne vorgelesene Wörter als auch Sätze aus den verschiedenen Versionen der Hamburger Schreibprobe (HSP; May, 2012) schreiben. Zur Erfassung der Rechtschreibkompetenz ist die HSP ein etabliertes und valides Verfahren. Die Wortauswahl orientierte sich dabei grundsätzlich an den Lehrplanzielen, sodass verschiedene Rechtschreibphänomene berücksichtigt wurden, wobei nicht ausschließlich Wörter aus Grund- oder Übungswortschätzen verwendet wurden. Auch die hohen Korrelationen der Ergebnisse der HSP mit der Einschätzung der Lehrpersonen sowie mit anderen Rechtschreibtests sprechen für eine gute Validität (May, 2012). Für die Auswertung der vorliegenden Untersuchung wurden nur rechtschreibschwierige Wörter verwendet, d.h. es wurden nur jene Wörter ausgewertet, bei denen sich die lautgetreue Schreibung von der orthographisch korrekten Schreibweise unterschied. Für $t 1$ wurden elf Wörter der HSP 1+ ausgewählt, für $t 2$ und $t 3$ konnten 17 Wörter mit orthographischen Schwierigkeiten der HSP 2 identifiziert werden. Die Reliabilitäten der Skalen fielen $\mathrm{zu}$ allen drei Messzeitpunkten zufriedenstellend aus $\left(\alpha_{t 1}=\right.$ $\left..68, \alpha_{t 2}=.88, \alpha_{t 3}=.84\right)$.

Kognitive Grundfähigkeit: $\mathrm{Zu} t 1$ wurden die kognitiven Fähigkeiten der Schülerinnen und Schüler mit Hilfe des Subtests Matrizen aus dem CFT-1 (Cattell, Weiß \& Osterland, 1997) erfasst. Dieser wurde als Wahrnehmungstest konstruiert, um möglichst sprachfrei die kognitiven Fähigkeiten zu erfassen. Der CFT-1 korreliert mit dem HAWIK-Intelligenztest auf hohem Niveau, hingegen nicht mit standardisierten Schulleistungstest und Schulnoten 
bzw. nur sehr gering, sodass die Validität des Tests als gegeben anzusehen ist (Cattell, Weiß \& Osterland, 1997). Dabei mussten die Schülerinnen und Schüler bei 12 Items jeweils das passende Muster aus fünf Antworten auswählen $(\alpha=.79)$. Mit dieser Skala sollte erfasst werden, inwiefern die Schülerinnen und Schüler in der Lage sind, „Regeln und Zusammenhänge bei figuralen Problemstellungen zu erkennen" (Cattell et al., 1997, S.5). Für jede Schülerin bzw. jeden Schüler wurde die Anzahl der richtig ausgewählten Antworten summiert, sodass die Werte entsprechend zwischen 0 und 12 rangieren konnten.

Arbeitsverhalten: Die Einschätzung der Ausdauer und der Konzentration erfolgte zu $t 1$ durch die Klassenlehrerinnen bzw. Klassenlehrer auf einer 4-stufigen LikertSkala von $0=$ Verhalten tritt nie auf bis $3=$ Verhalten tritt häufig auf mit Hilfe von je fünf Items aus der Lehrereinschätzliste (LSL; Petermann \& Petermann, 2006 z. B. „Der Schüler bzw. die Schülerin strengt sich an, um eine Aufgabe zu lösen."). Sparfeldt, Rost, Schleebusch und Heise (2012) konnten zeigen, dass dieses Instrument sowohl über konvergente Validität als auch über diskriminante Validität verfügt. Für die Analysen wurde für jede Schülerin bzw. jeden Schüler der Skalenmittelwert gebildet. Die Reliabilitäten fielen mit $\alpha=.91$ für Ausdauer und $\alpha=.94$ für Konzentration sehr gut aus und entsprechen den Reliabilitäten der Normstichprobe.

\section{Statistische Verfahren und Voranalysen}

Ein wesentlicher Schritt zur Beantwortung der Fragestellung war die Zuordnung der Kinder zu den Kompetenzniveaus. Dies war notwendig, da es sich bei den Niveaus theoretisch um qualitativ unterschiedliche Entwicklungsschritte handelt. Hierbei kommt es dazu, dass die Schülerinnen und Schüler zunächst immer mehr Wörter lautgetreu schreiben. Anschließend berücksichtigen die Schülerinnen und Schüler zusätzlich orthographische Regeln. Dadurch nimmt der Anteil der korrekt geschriebenen Wörter zu. Für beide Variablen ist demnach kein kontinuierlich linearer Verlauf zu erwarten. Um die unterschiedlichen Niveaus bei der Schreibung zu berücksichtigen, wurde für jedes Wort eine der folgenden drei Kategorien verwendet: orthographisch korrekt (2), lautgetreu (1) oder nicht lautgetreu (0). Um zu entscheiden, ob ein Wort lautgetreu verschriftet wurde, schätzten zwei unabhängige Raterinnen und Rater das Wort in Anlehnung an Moll und Landerl (2010) ein (Cohens Kappa $K_{t 1}=0.83, K_{t 2}$ $\left.=0.82, K_{t 3}=0.85\right)$. Anschließend wurde für jedes Kind berechnet, wie viel Prozent der Wörter die jeweilige Kodierung erhielten. $\mathrm{Zu}$ jedem Messzeitpunkt wurden pro Kind drei Prozentangaben gebildet. Diese neun Variablen bildeten die Analysevariablen der latenten Transitions- analyse in MPlus 6.0 (Muthén \& Muthén, 1988-2012), mit der die Kinder den Kompetenzniveaus zugeordnet wurden. Dieses Verfahren ist eine längsschnittliche Erweiterung der latenten Klassenanalyse, welches sich vor allem für Gruppeneinteilungen eignet, die sich qualitativ voneinander unterscheiden. Innerhalb einer Gruppe sollen dabei die Rechtschreibkompetenzen der Schülerinnen und Schüler möglichst homogen sein. Die Gruppen wurden dabei so gebildet, dass zu allen drei Messzeitpunkten inhaltlich gleiche Gruppen entstehen, wobei der Anteil der Schülerinnen und Schüler innerhalb einer Gruppe variieren konnte. Dadurch war es möglich, die Schülerinnen und Schüler zu jedem Messzeitpunkt einem der folgenden drei Profile zuzuordnen: Schülerinnen und Schüler im Profil Buchstabenentdeckende schreiben nur sehr wenige Wörter lautgetreu oder korrekt, hingegen den größten Anteil der Wörter noch nicht lautgetreu. Das Profil der Phonemprofis zeichnet sich dadurch aus, dass die Schülerinnen und Schüler den größten Anteil der Wörter lautgetreu schreiben und das Profil der Rechtschreibspezialisten durch überwiegend korrekte Schreibungen. Um den inhaltlichen Mehrwert der Profile zu verdeutlichen, soll folgendes Beispiel dienen: Sowohl Schülerinnen und Schüler im Profil der Buchstabenentdeckenden als auch im Profil der Phonemprofis könnten 20 \% der Wörter korrekt geschrieben haben. Sie unterscheiden sich jedoch darin, welche Strategie sie für die Verschriftung der anderen Wörter verwendet haben (Buchstabenentdeckende haben einen deutlich höheren Anteil an Wörtern, welche noch nicht lautgetreu verschriftet wurden). Nur die Berücksichtigung des quantitativen Maßes korrekt geschriebener Wörter würde damit wesentliche Unterschiede hinsichtlich des Prozesses der Rechtschreibkompetenzentwicklung nicht abdecken, sondern stattdessen nur anzeigen, wie viel Prozent des Zielzustandes erreicht wurden.

In Tabelle 1 ist die Verteilung der Schülerinnen und Schüler in den Profilen zu den jeweiligen Messzeitpunkten dargestellt. Hierbei wird deutlich, dass zu $t 1$ die meisten Schülerinnen und Schüler dem Profil der Phonemprofis zugeordnet wurden. Ein kleiner Teil der Schülerinnen und Schüler konnte schon zu Beginn der zweiten Klasse die meisten Wörter orthographisch korrekt schreiben, wohingegen $11 \%$ dem Profil der Buchstabenentdeckenden zugewiesen wurden. Die Verteilung ändert sich zu $t 2$ und $t 3$ insofern, dass der Anteil in den Profilen Buchstabenentdeckende und Phonemprofis abnimmt, wohingegen die Anzahl der Schülerinnen und Schüler im Profil der Rechtschreibspezialisten stark ansteigt. Nichtsdestotrotz verharren einige Schülerinnen und Schüler auf dem Profil der Buchstabenentdeckenden bzw. der Phonemprofis (weitere Informationen finden sich in Jaeuthe et al., 2020).

In Tabelle 2 zeigen sich hohe Korrelationen für die Rechtschreibleistung, gemessen als der Anteil der richtig 
Tabelle 1. Anteil der Profile an der Gesamtstichprobe, Anteil der Mädchen in den Profilen und deskriptive Kennwerte

\begin{tabular}{|c|c|c|c|c|c|c|c|c|}
\hline \multirow[t]{2}{*}{ Profil } & \multicolumn{3}{|c|}{ Anteil Stichprobe in \% } & \multicolumn{3}{|c|}{ Anteil Mädchen in \% } & \multirow[t]{2}{*}{ Kognitive Grundfähigkeit $M(S D)$} & \multirow{2}{*}{$\begin{array}{c}\text { Arbeitsverhalten } \\
\qquad M(S D)\end{array}$} \\
\hline & $t 1$ & t2 & t3 & $t 1$ & t2 & t3 & & \\
\hline Buchstabenentdeckende & 11.35 & 8.55 & 5.92 & 34.78 & 40.38 & 33.33 & $6.61(2.73)$ & $1.45(0.67)$ \\
\hline Phonemprofis & 80.10 & 58.72 & 17.27 & 52.16 & 46.78 & 41.90 & $9.11(2.63)$ & $2.26(0.62)$ \\
\hline Rechtschreibspezialisten & 8.55 & 32.73 & 76.81 & 57.69 & 60.30 & 53.96 & $9.88(2.72)$ & $2.68(0.38)$ \\
\hline
\end{tabular}

Anmerkungen: Kognitive Grundfähigkeit und Arbeitsverhalten wurden nur zum ersten Messzeitpunkt erhoben.

Tabelle 2. Bivariate Korrelationen zwischen der Rechtschreibleistung und den individuellen Merkmalen

\begin{tabular}{|c|c|c|c|c|c|}
\hline & (1) & (2) & (3) & (4) & (5) \\
\hline (1) Rechtschreibleistung t1 & - & & & & \\
\hline (2) Rechtschreibleistung t2 & .68 & - & & & \\
\hline (3) Rechtschreibleistung t3 & .56 & .75 & - & & \\
\hline (4) Geschlecht & -.17 & -.17 & -.17 & - & \\
\hline (5) kognitive Grundfähigkeiten & .27 & .26 & .25 & -.08 & - \\
\hline (6) Arbeitsverhalten & .42 & .54 & .51 & -.21 & .31 \\
\hline
\end{tabular}

Anmerkungen: Für die Rechtschreibleistung wurde hier der Anteil der richtig geschriebenen Wörter berücksichtigt; kognitive Grundfähigkeit und Arbeitsverhalten wurden nur zum ersten Messzeitpunkt erhoben. Alle Korrelationen sind auf dem Niveau $p<.05$ signifikant. Geschlecht: $0=$ weiblich, $1=$ männlich.

geschriebenen Wörter, über die drei Messzeitpunkte hinweg. Hingegen zeigen sich nur schwache Zusammenhänge zwischen dem Geschlecht und den Rechtschreibleistungen, den kognitiven Fähigkeiten und dem Arbeitsverhalten. Die kognitiven Fähigkeiten und das Arbeitsverhalten korrelieren mit $r=.31$ auf mittlerem Niveau.

Zur Beantwortung der Forschungsfragen wurden sodann multinomiale logistische Regressionen in SPSS berechnet. Hierfür wurden die Zuordnungen zu den Profilen für jeden Messzeitpunkt als Variablen in SPSS gespeichert. Zusätzlich wurden zwei Variablen erstellt, in denen die Wechsel zwischen den Messzeitpunkten codiert wurden (z.B. $0=$ zu $t 1$ und $t 2$ Buchstabenentdeckende, $1=$ Wechsel von Buchstabenentdeckende zu Phonemprofis von $t 1 \mathrm{zu} t 2$ ). Anschließend wurden die Variablen Geschlecht, kognitive Grundfähigkeit und Arbeitsverhalten als Kovariaten schrittweise in die Modelle eingefügt. Als Referenzgruppe wurde dabei das Profil der Phonemprofis verwendet, welches dem mittleren Kompetenzniveau entspricht. Im ersten Modell wird der Zusammenhang mit dem Geschlecht geprüft. Anschließend wurde zusätzlich die kognitive Grundfähigkeit der Schülerinnen und Schüler berücksichtigt (Modell 2). Im letzten Modell (Modell 3) wurde schließlich geprüft, inwiefern sich der Zusammenhang der Rechtschreibkompetenz mit dem Geschlecht und der kognitiven Grundfähigkeit verändert, wenn zusätzlich das Arbeitsverhalten in das Modell aufgenommen wurde. Für jedes Modell werden die aufgeklärte Varianz, Odds und Odd-Ratios angegeben. Da in den multiplen
Regressionen viele Modelle an einer Stichprobe berechnet werden, wurde ein konservatives Signifikanzniveau von .01 gewählt, um das Risiko von Alpha-Fehler-Inflationierungen zu minimieren.

Bei der Prüfung der Voraussetzungen für die logistische Regression fiel auf, dass die Variablen Ausdauer und Konzentration sehr hoch korrelierten $(r=.87)$. Aus diesem Grund wurde mit Hilfe der Kollinearitätsdiagnostik geprüft, inwiefern die beiden Variablen als Kovariaten jeweils unterschiedliche Anteile der Varianz aufklärten. Hierbei zeigten sich hohe Überschneidungen der Varianzanteile. Dies widerspricht der Voraussetzung der Multikollinearität, sodass für die Regressionsanalysen eine neue Variable („Arbeitsverhalten“) als Mittelwert aus den Variablen Ausdauer und Konzentration gebildet wurde.

Mit Hilfe der standardisierten Residuen wurde geprüft, inwiefern Fälle auftraten, die nicht gut zum Modell passen. In Anlehnung an Stevens (2002) wurde für diese Fälle mit Hilfe der Cook's distance geprüft, ob es sich um einflussreiche Fälle handelt. Da für alle auftretenden Fälle $(n=11)$ die Cook's distance deutlich unter dem geforderten Wert von 1 lag, verblieben die Fälle in der Stichprobe.

Als letzte Voraussetzung wurde die Linearität des Logits untersucht, indem geprüft wurde, ob die Interaktion zwischen den Kovariaten und deren log-Transformationen in einem nicht-linearen Zusammenhang mit der abhängigen Variable stehen. Die Interaktion fiel lediglich für eine Variable, dem Arbeitsverhalten, und nur zu t3 signi- 
fikant aus, sodass diese Ergebnisse mit entsprechender Vorsicht interpretiert werden müssen.

\section{Ergebnisse}

Zunächst wird an dieser Stelle auf die deskriptiven Kennwerte eingegangen. In Tabelle 1 sind für jeden Messzeitpunkt der Anteil Mädchen in den Profilen dargestellt. Hierbei wird deutlich, dass zu jedem Messzeitpunkt mehr Mädchen als Jungen im Profil der Rechtschreibspezialisten und zugleich weniger Mädchen als Jungen im Profil der Buchstabenentdeckenden zu finden waren. Zudem zeigten sich auf deskriptiver Ebene Unterschiede zwischen den Profilen dahingehend, dass die Buchstabenentdeckenden über die geringsten kognitiven Fähigkeiten verfügten, aber auch im Arbeitsverhalten die geringsten Werte zeigten. Hingegen wiesen die Rechtschreibspezialisten in beiden Merkmalen die höchste Ausprägung auf.

In Tabelle 3 ist dargestellt, inwiefern die Kovariaten mit der Zuordnung zu einem der Profile zusammenhängen. Diese Ergebnisse werden für jeden der drei Messzeitpunkte separat dargestellt. Um Geschlechterunterschiede zu prüfen, wurde im ersten Modell zunächst nur das Geschlecht in die Regressionsanalysen einbezogen (s. Modell 1, H1). Es zeigte sich, dass Mädchen signifikant wahrscheinlicher den höheren Profilen zugeordnet wurden. Dies betrifft zu $t 1$ den Vergleich zwischen den Buchstabenentdeckenden, wohingegen zu $t 2$ der Vergleich zwischen den Phonemprofis und den Rechtschreibspezialisten signifikant wird. Die Varianzaufklärung für dieses Modell fällt mit $R^{2}=.02$ sehr gering aus.

Im nächsten Schritt wurde geprüft, ob Schülerinnen und Schüler mit hohen kognitiven Grundfähigkeiten höheren Kompetenzniveaus zugeordnet werden (H2). Hierfür wurden die Regressionsanalysen im Modell 2 um die kognitive Grundfähigkeit erweitert. Zusammenhänge mit dem Geschlecht blieben nur zu $t 2$ bestehen. Darüber hinaus hing die kognitive Grundfähigkeit zu allen drei Messzeitpunkten mit der Zuordnung zu den Profilen zusammen. Schülerinnen und Schüler mit einer niedrigeren kognitiven Grundfähigkeit wurden signifikant wahrscheinlicher dem niedrigsten Profil, den Buchstabenentdeckenden, zugeordnet als dem der Phonemprofis. Schülerinnen und Schüler mit hoher kognitiver Grundfähigkeit wurden im Vergleich zum mittleren Profil signifikant wahrscheinlicher dem höchsten Profil, den Rechtschreibspezialisten, zugeordnet. In Modell 2 stieg die Varianzaufklärung $\left(R_{t 1}{ }^{2}=.13 ; R_{t 2}{ }^{2}=.09 ; R_{t 3}{ }^{2}=.08\right)$.

Im dritten Schritt wurden zusätzlich die Effekte des Arbeitsverhaltens betrachtet, um zu ermitteln, inwiefern Schülerinnen und Schüler mit positivem Arbeitsverhalten eine höhere Wahrscheinlichkeit haben, höheren Profilen zugeordnet zu werden (H3, Modell $3 \mathrm{zu} t 1, t 2$ und $t 3$ ). Der Zusammenhang der Rechtschreibkompetenz mit dem Arbeitsverhalten fiel zu allen drei Messzeitpunkten signifikant aus. Unter Kontrolle des Arbeitsverhaltens hatte die kognitive Grundfähigkeit nur zu $t 1$ beim Vergleich der Buchstabenentdeckenden und der Phonemprofis einen signifikant eigenständigen Effekt, der Zusammenhang mit dem Geschlecht wurde zu keinem Messzeitpunkt signifikant. Das Arbeitsverhalten der Schülerinnen und Schüler konnte die Zuordnung zu den Profilen signifikant vorhersagen. Steigt das Arbeitsverhalten der Schülerinnen und Schüler um eine Einheit, so hatten diese eine sechsmal $(t 1)$, fünfmal ( $t 2)$ bzw. fast dreimal ( $t 3)$ höhere Wahrscheinlichkeit dem Profil der Rechtschreibspezialisten zugeordnet zu werden als den Phonemprofis. Im Modell 3 fällt die Varianzaufklärung mit $R^{2}=.23 \mathrm{zu} t 3$ bis $R^{2}=.29 \mathrm{zu}$ $t 1$ am höchsten aus.

Um die Entwicklungshypothesen (H4, H5) zu prüfen, wurden weitere logistische Regressionen berechnet, deren Ergebnisse in den Tabellen 4 und 5 dargestellt sind. Bezüglich der Wechsel vom Profil der Buchstabenentdeckenden zum Profil der Phonemprofis konnten keine signifikanten Zusammenhänge gefunden werden (Tabelle 4). Hingegen hing das Arbeitsverhalten mit höheren Wahrscheinlichkeiten zusammen, um vom Profil der Phonemprofis zum Profil der Rechtschreibspezialisten zu wechseln (Tabelle 5) ( $t 1 \mathrm{zu} t 2: R^{2}=.32$ bzw. $\left.t 2 \mathrm{zu} t 3: R^{2}=.29\right)$.

\section{Diskussion}

\section{Zusammenfassung der Ergebnisse}

In der vorliegenden Studie sollte der Zusammenhang der individuellen Merkmale Geschlecht, kognitive Grundfähigkeit und Arbeitsverhalten mit der Entwicklung der Rechtschreibkompetenz längsschnittlich untersucht werden. Dabei wurden Zusammenhänge sowohl mit der $\mathrm{Zu}-$ ordnung der Schülerinnen und Schüler zu einem der Rechtschreibkompetenzniveaus als auch mit den Wechseln von einem niedrigerem zum nächsthöheren Niveau berücksichtigt. Die erste Hypothese (H1) bezog sich darauf, dass Mädchen schon früher höhere Kompetenzniveaus erreichen. Hier wurden hypothesenkonform signifikante Zusammenhänge mit der Zuordnung zu den Profilen gefunden. Jedoch verschwinden diese, sobald das Arbeitsverhalten berücksichtigt wurde. Die Ergebnisse sprechen daher eher für ein unterschiedliches Arbeitsverhalten der Mädchen und Jungen, welches zu den Unterschieden hinsichtlich der Rechtschreibkompetenz führt. Dies deckt sich mit den Ergebnissen von Machts 
Tabelle 3. Koeffizienten der schrittweisen logistischen Regression mit Geschlecht, kognitiver Grundfähigkeit und Arbeitsverhalten als Prädiktoren und der Gruppe der Phonemprofis als Referenzgruppe

\begin{tabular}{|c|c|c|c|c|c|c|c|c|c|c|}
\hline \multirow[b]{2}{*}{ Profil } & \multirow[b]{2}{*}{ Effekt } & \multicolumn{3}{|c|}{ Modell 1} & \multicolumn{3}{|c|}{ Modell 2} & \multicolumn{3}{|c|}{ Modell 3} \\
\hline & & $\beta$ & SE & OR & $\beta$ & SE & OR & $\beta$ & SE & OR \\
\hline \multicolumn{11}{|l|}{ t1 } \\
\hline \multirow[t]{3}{*}{ Buchstabenentdeckende } & Geschlecht & $.72 *$ & .27 & 2.05 & .73 & .29 & 2.06 & .34 & .31 & 1.40 \\
\hline & kog. Fähig. & - & - & - & $-.30 *$ & .05 & .74 & $-.23^{*}$ & .05 & .79 \\
\hline & Arbeitsverh. & - & - & - & - & - & - & $-1.48 *$ & .23 & .23 \\
\hline \multirow[t]{3}{*}{ Rechtschreibspezialisten } & Geschlecht & -.22 & .30 & .80 & -.17 & .30 & .84 & .09 & .31 & 1.10 \\
\hline & kog. Fähigk. & - & - & - & .13 & .06 & 1.13 & .05 & .07 & 1.05 \\
\hline & Arbeitsverh. & - & - & - & - & - & - & $1.79 *$ & .42 & 6.02 \\
\hline$R^{2}$ & & .02 & - & - & .13 & - & - & .29 & - & - \\
\hline \multicolumn{11}{|l|}{$t 2$} \\
\hline \multirow[t]{3}{*}{ Buchstabenentdeckende } & Geschlecht & .26 & .30 & 1.30 & .28 & .32 & 1.33 & -.03 & .33 & .97 \\
\hline & kog. Fähigk. & - & - & - & $-.15^{\star}$ & .05 & .86 & -.09 & .05 & .91 \\
\hline & Arbeitsverh. & - & - & - & - & - & - & $-1.10 *$ & .24 & .33 \\
\hline \multirow[t]{3}{*}{ Rechtschreibspezialisten } & Geschlecht & $-.56^{\star}$ & .18 & .57 & $-.56 *$ & .19 & .57 & -.30 & .20 & .75 \\
\hline & kog. Fähigk. & - & - & - & $.14^{\star}$ & .04 & 1.15 & .06 & .04 & 1.06 \\
\hline & Arbeitsverh. & - & - & - & - & - & - & $1.65^{\star}$ & .22 & 5.21 \\
\hline$\overline{R^{2}}$ & & .02 & - & - & .09 & - & - & .28 & - & - \\
\hline \multicolumn{11}{|l|}{ t3 } \\
\hline \multirow[t]{3}{*}{ Buchstabenentdeckende } & Geschlecht & .37 & .41 & 1.44 & .45 & .43 & 1.58 & .18 & .45 & 1.20 \\
\hline & kog. Fähigk. & - & - & - & -.14 & .07 & .87 & -.09 & .07 & .91 \\
\hline & Arbeitsverh. & - & - & - & - & - & - & $-.95 *$ & .31 & .39 \\
\hline \multirow[t]{3}{*}{ Rechtschreibspezialisten } & Geschlecht & -.49 & .22 & .62 & -.48 & .22 & .62 & -.23 & .24 & .80 \\
\hline & kog. Fähigk. & - & - & - & $.12^{\star}$ & .04 & 1.13 & .06 & .04 & 1.06 \\
\hline & Arbeitsverh. & - & - & - & - & - & - & $1.05^{\star}$ & .18 & 2.85 \\
\hline$\overline{R^{2}}$ & & .02 & - & - & .08 & - & - & .23 & - & - \\
\hline
\end{tabular}

Anmerkungen: $\beta=$ Regressionskoeffizient, $S E=$ Standardfehler, $\mathrm{OR}=$ Odds Ratio, Geschlecht: 0 = weiblich, $1=$ männlich, kog. Fähigk. = kognitive Grundfähigkeit, Arbeitsv. $=$ Arbeitsverhalten, ${ }^{\star} p<.01$.

Tabelle 4. Schrittweise logistische Regression der Wechsel vom Profil der Buchstabenentdeckenden zum Profil Phonemprofis mit der Gruppe, die auf dem Profil der Buchstabenentdeckenden verharrt als Referenzgruppe

\begin{tabular}{|c|c|c|c|c|c|c|c|c|c|c|}
\hline \multirow[b]{2}{*}{ Messzeitpunkt des Wechsels } & \multirow[b]{2}{*}{ Effekt } & \multicolumn{3}{|c|}{ Modell 1} & \multicolumn{3}{|c|}{ Modell 2} & \multicolumn{3}{|c|}{ Modell 3} \\
\hline & & $\beta$ & SE & $\mathrm{OR}$ & $\beta$ & SE & OR & $\beta$ & SE & $\mathrm{OR}$ \\
\hline \multirow[t]{3}{*}{ t1 zu t2 } & Geschlecht & .54 & .57 & 1.72 & .44 & .58 & 1.56 & .50 & .59 & 1.64 \\
\hline & kog. Fähigk & - & - & - & -.13 & .09 & .88 & -.13 & .09 & .88 \\
\hline & Arbeitsverh. & - & - & - & - & - & - & .11 & .40 & 1.11 \\
\hline$R^{2}$ & & .03 & & & .14 & & & .32 & & \\
\hline \multirow[t]{3}{*}{ t2 zu t3 } & Geschlecht & -.09 & .68 & .91 & -.18 & .69 & .84 & .04 & .71 & 1.04 \\
\hline & kog. Fähigk & - & - & - & .16 & .11 & 1.17 & .13 & .12 & 1.14 \\
\hline & Arbeitsverh. & - & - & - & - & - & - & .78 & .50 & 2.18 \\
\hline$\overline{R^{2}}$ & & .03 & - & - & .10 & - & - & .29 & - & - \\
\hline
\end{tabular}

Anmerkungen: $\beta=$ Regressionskoeffizient, $S E=$ Standardfehler, $\mathrm{OR}=$ Odds Ratio, Geschlecht: $0=$ weiblich, $1=$ männlich kog. Fähigk. $=$ kognitive Grundfähigkeit, Arbeitsv. $=$ Arbeitsverhalten .

und Möller (2019), die ebenfalls überfachliche Kompetenzen als ausschlaggebend für Geschlechterunterschiede hinsichtlich der Deutschnote und der Testleistung im Fachbereich Deutsch ausfindig machten. Diese Annahme konnte auf Grund der vorliegenden Studie durch die Berücksichtigung der Lernermöglicher (DiPerna, 2006) konkretisiert werden. Mädchen und Jungen unterscheiden sich hinsichtlich ihrer Anstrengungsbereitschaft und 
Tabelle 5. Logistische Regression der Wechsel vom Profil der Phonemprofis zum Profil Rechtschreibspezialisten mit der Gruppe, die auf dem Profil der Phonemprofis verbleibt als Referenzgruppe

\begin{tabular}{|c|c|c|c|c|c|c|c|c|c|c|}
\hline \multirow[b]{2}{*}{ Messzeitpunkt des Wechsels } & \multirow[b]{2}{*}{ Effekt } & \multicolumn{3}{|c|}{ Modell 1} & \multicolumn{3}{|c|}{ Modell 2} & \multicolumn{3}{|c|}{ Modell 3} \\
\hline & & $\beta$ & SE & OR & $\beta$ & SE & OR & $\beta$ & SE & OR \\
\hline \multirow[t]{3}{*}{ t1 zu t2 } & Geschlecht & $-.54^{\star}$ & .20 & .59 & $-.57 *$ & .21 & .57 & -.34 & .22 & .71 \\
\hline & kog. Fähigk & - & - & - & $.11 *$ & .04 & 1.12 & .05 & .04 & 1.05 \\
\hline & Arbeitsverh. & - & - & - & - & - & - & $1.41 *$ & .24 & 4.11 \\
\hline$\overline{R^{2}}$ & & .03 & & & .14 & & & .32 & & \\
\hline \multirow[t]{3}{*}{ t2 zu t3 } & Geschlecht & -.26 & .25 & .29 & -.26 & .25 & .78 & -.12 & .26 & .89 \\
\hline & kog. Fähigk & - & - & - & .07 & .04 & 1.07 & .04 & .05 & 1.04 \\
\hline & Arbeitsverh. & - & - & - & - & - & - & $.59 *$ & .20 & 1.80 \\
\hline$\overline{R^{2}}$ & & .03 & - & - & .10 & - & - & .29 & - & - \\
\hline
\end{tabular}

Anmerkungen: $\beta=$ Regressionskoeffizient, $S E=$ Standardfehler, $\mathrm{OR}=$ Odds Ratio, Geschlecht: $0=$ weiblich, $1=$ männlich kog. Fähigk. $=$ kognitive Grundfähigkeit, Arbeitsverh. $=$ Arbeitsverhalten, ${ }^{\star} p<.01$.

Ausdauer und diese Merkmale wiederum konnten Unterschiede hinsichtlich der Rechtschreibkompetenzniveaus erklären.

Die Zuordnung der Schülerinnen und Schüler zu einem Niveau hing außerdem mit der kognitiven Grundfähigkeit zusammen (H2). Jedoch fanden sich kaum Zusammenhänge mit den Wechseln zwischen den Profilen (H4). Die Ergebnisse decken sich mit den Befunden von Stranghöner et al. (2017). Auch dort zeigten sich keine Einflüsse der kognitiven Grundfähigkeit auf die Entwicklung der Rechtschreibleistung der Kinder. Stranghöner et al. (2017) schlussfolgerten, dass stattdessen domänenspezifische Vorkenntnisse für die Entwicklung ausschlaggebend sein könnten. Eine andere Erklärung könnte jedoch darin liegen, dass Schülerinnen und Schüler mit höheren kognitiven Grundfähigkeiten auch ein besseres Arbeitsverhalten zeigen und sich deshalb der Effekt der kognitiven Grundfähigkeit reduziert. So konnte in der vorliegenden Studie gezeigt werden, dass der Zusammenhang der kognitiven Grundfähigkeit unter Berücksichtigung des Arbeitsverhaltens keinen eigenständigen signifikanten Zusammenhang zeigte. Die vorliegenden Befunde unterstreichen damit die Bedeutsamkeit des Arbeitsverhaltens für die Entwicklung der Rechtschreibkompetenz.

Wie in $\mathrm{H} 3$ vermutet, zeigten sich Zusammenhänge zwischen dem Arbeitsverhalten und der Zuordnung zu einem Profil. Je positiver das Arbeitsverhalten der Schülerinnen und Schüler ausfiel, desto höher war auch die Wahrscheinlichkeit, dem höchsten Kompetenzniveau zugeordnet zu werden. Somit konnte der von Kikas und Silinskas (2016) bereits gefundene positive Einfluss des Arbeitsverhaltens nun auch für die Rechtschreibkompetenzniveaus bestätigt werden. Auffällig ist jedoch, dass die Effekte für die Zuordnung zum Profil der Rechtschreibspezialisten im Vergleich zu den Phonemprofis deutlich höher ausfielen als die Effekte beim Vergleich der
Buchstabenentdeckenden zu den Phonemprofis. Dies könnte daraufhin deuten, dass das Arbeitsverhalten eher für das Erreichen höherer Kompetenzniveaus notwendig ist, wohingegen das Arbeitsverhalten beim Erreichen des Profils der Phonemprofis eine geringere, aber dennoch signifikante Rolle spielt. Hierfür sind verschiedene Erklärungen denkbar. Wenn Schülerinnen und Schüler über ein positives Arbeitsverhalten verfügen, haben diese mehr Erfolg beim Lernen, sodass dies langfristig zu einer positiven Leistungsentwicklung führt (DiPerna, 2006). Demgegenüber könnten Schülerinnen und Schüler mit einem weniger positiven Arbeitsverhalten bei schwierigen Aufgaben eher aufgeben, wodurch sich die Erwartung verfestigen kann, bei Aufgaben zu scheitern (Georgiou et al., 2010). Andersherum könnten jedoch gerade Schülerinnen und Schüler, welche Schwierigkeiten beim Rechtschreiberwerb haben, eine geringere Ausdauer zeigen, da für sie das Schreiben unbekannter Wörter zu schwierig und unangenehm ist. Dies könnte auch erklären, wieso das Arbeitsverhalten insbesondere für Wechsel vom Profil der Phonemprofis zum Profil der Rechtschreibspezialisten bedeutsam war, nicht jedoch für Wechsel vom Profil der Buchstabenentdeckenden zum Profil der Phonemprofis (H5). So scheint es für den Großteil der Schülerinnen und Schüler wenig problematisch zu sein, die Lautfolge der Wörter zu entschlüsseln. Für das Erlernen von orthographischen Regeln, die über eine lautgetreue Schreibung hinausgehen, wird hingegen eine höhere Ausdauer und Konzentration benötigt. Weiterhin ist denkbar, dass für den Wechsel von nicht lautgetreuem zu lautgetreuem Schreiben andere Merkmale, wie z.B. die phonologische Bewusstheit ausschlaggebend sein könnten.

Zusammenfassend verdeutlichen die Befunde die Relevanz des Arbeitsverhaltens für die Entwicklung der Rechtschreibkompetenz von lautgetreuen Schreibungen hin zu orthographisch korrektem Schreiben. Dabei konnte 
die vorliegende Studie Hinweise dafür liefern, dass die in bisherigen Studien gefundenen Geschlechterunterschiede auf ein unterschiedliches Arbeitsverhalten der Mädchen und Jungen zurückgeführt sein könnten.

\section{Limitationen und Ausblick}

Eine erste Limitation liegt in der Auswahl der Einflussfaktoren auf die Rechtschreibkompetenz. In der vorliegenden Studie wurden ausschließlich unspezifische Einflussfaktoren berücksichtigt. Dies lag darin begründet, dass diese Studie Teil eines größeren Projektes war, in dem es darum ging, schulnahe Prädiktoren für unterschiedliche schulische Leistungen im Verlauf der Grundschulzeit zu erfassen. Aus diesem Grund lag der Fokus der vorliegenden Studie auf im schulischen Kontext veränderbare fächerübergreifende Merkmale. Dies hat jedoch zur Folge, dass keine Aussagen darüber getroffen werden können, inwiefern die Bedeutung der unspezifischen Einflussmerkmale unter Kontrolle von spezifischen Merkmalen zu denselben Ergebnissen geführt hätte. Hinzu kommt, dass die unspezifischen Merkmale (Arbeitsverhalten und kognitive Grundfähigkeit) nur zu $t 1$ erhoben wurden. Dadurch sind einerseits keine wechselseitigen Veränderungen abbildbar und andererseits sind keine Aussagen darüber möglich, inwiefern das Arbeitsverhalten sich im Laufe der Studie verändert haben könnte. In zukünftigen Untersuchungen sollten diese Variablen zu allen Messzeitpunkten erhoben werden. Hierbei muss auch berücksichtigt werden, dass ursprünglich zwei $\mathrm{Fa}$ cetten des Arbeitsverhaltens, die Ausdauer und die Konzentration, berücksichtigt werden sollten. Im Ergebnis zeigten sich sehr hohe Korrelationen, sodass die beiden Merkmale für die weiteren Analysen zusammengefasst werden mussten. In zukünftigen Studien sollten die Einschätzungen der Lehrpersonen mit weiteren Testverfahren kombiniert werden.

Eine weitere Limitation liegt in der Operationalisierung der kognitiven Grundfähigkeit der Schülerinnen und Schüler. Hier wurde die Skala Matrizen des CFT-1 (Cattell et al., 1997) verwendet und die Anzahl der richtig gelösten Aufgaben in die Analysen einbezogen. Es handelt sich hierbei demnach nicht um einen standardisierten Intelligenzquotienten, sondern lediglich um eine Annäherung an die kognitiven Grundfähigkeiten der Schülerinnen und Schüler. Hier könnten in zukünftigen Untersuchungen unterschiedliche Facetten von Intelligenz erfasst werden, um z.B. auch sprachgebundene Anteile von Intelligenz in Beziehung zum Arbeitsverhalten und zur Rechtschreibkompetenz zu setzen.

Im vorliegenden Beitrag konnte der Zusammenhang des Arbeitsverhaltens mit der Rechtschreibkompetenz der Schülerinnen und Schüler beim Schreiben von diktierten Wörtern gezeigt werden. Offen bleibt hingegen, inwiefern weitere Schreibfacetten, wie z. B. das Schreiben von Texten, von individuellen Merkmalen der Schülerinnen und Schülern beeinflusst werden. Zukünftige Studien mit älteren Zielgruppen könnten daher in den Blick nehmen, inwiefern diese Kompetenzen auch durch das Arbeitsverhalten der Schülerinnen und Schüler beeinflusst werden.

\section{Pädagogische Implikationen}

Die vorliegende Längsschnittstudie liefert Hinweise darauf, dass das Arbeitsverhalten der Schülerinnen und Schüler einen bedeutsamen Einfluss auf die Entwicklung der Rechtschreibkompetenz von lautgetreuem zu orthographisch korrektem Schreiben hat. Basierend auf diesen Befunden sollte daher in weiterführenden Studien überprüft werden, inwiefern entsprechend ausgerichtet pädagogische Interventionen effektiv sind. So könnte es für Lehrpersonen sinnvoll sein, das Arbeitsverhalten der Schülerinnen und Schüler, die Schwierigkeiten bei diesem Wechsel zeigen, kontinuierlich zu beobachten und durch passende Methoden zu fördern. Darüber hinaus könnte dadurch überprüft werden, inwiefern kontinuierliches Feedback zu einer Steigerung des Arbeitsverhaltens der Schülerinnen und Schülern führt und inwiefern dies zu einer Verbesserung der Rechtschreib- und anderen schulischen Kompetenzen beiträgt. Hierbei sollte vor allem das Arbeitsverhalten der Jungen berücksichtigt werden. Diese Implikationen beziehen sich ausschließlich auf die in der vorliegenden Studie untersuchten Merkmale. Für eine umfassende Förderung der Rechtschreibkompetenz müssen Lehrpersonen auch andere relevante Merkmale berücksichtigen. 


\section{Literatur}

Baumert, J., Blum, W. \& Neubrand, M. (2004). Drawing the lessons from PISA 2000 - Long-term research implications: Gaining a better understanding of the relationship between system inputs and learning outcomes by assessing instructional and learning processes as mediating factors. In D. Lenzen, J. Baumert, R. Watermann \& U. Trautwein (Hrsg.), PISA und die Konsequenzen für die erziehungswissenschaftliche Forschung. (S. 143 -157). Wiesbaden: Verlag für Sozialwissenschaften.

Brügelmann, H. \& Brinkmann, E. (1994). Stufen des Schriftspracherwerbs und Ansätze zu seiner Förderung. In H. Brügelmann \& S. Richter (Hrsg.), Wie wir recht schreiben lernen: 10 Jahre Kinder auf dem Weg zur Schrift (S. 44-52). Lengwil: Libelle.

Bulut, N. (2018). Individuelle Rechtschreibentwicklung: Eine Längsschnittuntersuchung zur Bedeutung von Einflussfaktoren auf die Wortschreibung (Dissertation, Universität zu Köln). Baltmannsweiler: Schneider Verlag Hohengehren.

Cattell, R. B., Weiß, R. H. \& Osterland, J. (1997). CFT 1-R:Grundintelligenzstest Skala 1 - Handanweisung für die Durchführung, Auswertung und Interpretation (5., Aufl.). Göttingen: Hogrefe.

Cattell, R. B. \& Horn, J. L. (1978). A check on the theory of fluid and crystallized intelligence with description of new subtest designs. Journal of Educational Measurement, 15, 139-164.

DiPerna, J. C. (2006). Academic enablers and student achievement: Implications for assessment and intervention services in the schools. Psychology in the Schools, 43, 7-17.

Dummert, F., Endlich, D., Schneider, W. \& Schwenck, C. (2014). Entwicklung schriftsprachlicher und mathematischer Leistungen bei Kindern mit und ohne Migrationshintergrund. Zeitschrift für Entwicklungspsychologie und Pädagogische Psychologie, 46, 115-132. https://doi.org/10.1026/0049-8637/a000110

Ennemoser, M., Marx, P., Weber, J. \& Schneider, W. (2012). Spezifische Vorläuferfertigkeiten der Lesegeschwindigkeit, des Leseverständnisses und des Rechtschreibens: Evidenz aus zwei Längsschnittstudien vom Kindergarten bis zur 4. Klasse. Zeitschrift für Entwicklungspsychologie und Pädagogische Psychologie, 44, 53 -67. https://doi.org/10.1026/0049-8637/a000057

Georgiou, G. K., Manolitsis, G., Nurmi, J.-E. \& Parrila, R. (2010). Does task-focused versus task-avoidance behavior matter for literacy development in an orthographically consistent language? Contemporary Educational Psychology, 35, 1-10. https:// doi.org/10.1016/j.cedpsych.2009.07.001

Goldammer, A. von, Mähler, C., Bockmann, A.-K. \& Hasselhorn, M. (2010). Vorhersage früher Schriftsprachleistungen aus vorschulischen Kompetenzen der Sprache und der phonologischen Informationsverarbeitung. Zeitschrift für Entwicklungspsychologie und Pädagogische Psychologie, 42, 48-56. https:// doi.org/10.1026/0049-8637/a000005

Jaeuthe, J., Lambrecht, J., Bosse, S., Bogda, K. \& Spörer, N. (2020). Entwicklung der Rechtschreibkompetenz im zweiten und dritten Schuljahr: Eine latente Transitionsanalyse zur Überprüfung theoretischer Annahmen. Zeitschrift für Erziehungswissenschaft, 23, $823-846$.

Kaufmann, A. (2007). Merkmale und Einstellungen von Schülern. In H. Ditton (Hrsg.), Kompetenzaufbau und Laufbahnen im Schulsystem: Ergebnisse einer Längsschnittuntersuchung an Grundschulen (S. 117-143). Münster: Waxmann.

Kikas, E. \& Silinskas, G. (2016). Task persistence mediates the effect of children's literacy skills on mother's academic help. Educational Psychology, 36, 975 -991. https://doi.org/10.1080/ 01443410.2015 .1045836

KMK, Kultusministerkonferenz (2005). Bildungsstandards im Fach Deutsch für den Primarbereich: Beschluss vom 15.10.2004.
Verfügbar unter: https://www.kmk.org/fileadmin/veroeffentlichungen_beschluesse/2004/2004_10_15-BildungsstandardsDeutsch-Primar.pdf

Machts, N. \& Möller, J. (2019). Geschlechterunterschiede auf Kompetenzrastern: Die Relevanz überfachlicher Kompetenzen. Zeitschrift für Entwicklungspsychologie und Pädagogische Psychologie, 51, 97 -109. https://doi.org/10.1026/0049-8637/a00 0211

Marx, P. (2007). Lese- und Rechtschreiberwerb. Paderborn: Ferdinand Schöningh.

May, P. (2012). HSP 1-10 Hamburger Schreib-Probe: Manual Handbuch - Diagnose orthographischer Kompetenz zur Erfassung der grundlegenden Rechtschreibkompetenzen. Stuttgart: Ernst Klett.

Moll, K. \& Landerl, K. (2010). Lese- und Rechtschreibtest: SLRT-II Weiterentwicklung des Salzburger Lese- und Rechtschreibtests (SLRT). Bern: Huber.

Muthén, L. K. \& Muthén, B. O. (2012). Mplus User's Guide: Seventh Edition. Los Angeles, CA: Muthén \& Muthén.

Petermann, U. \& Petermann, F. (2006). Lehrereinschätzliste für Sozial- und Lernverhalten (LSL): Manual. Göttingen: Hogrefe.

Pfost, M. (2015). Children's phonological awareness as a predictor of reading and spelling: A systematic review of longitudinal research in German-speaking countries. Zeitschrift für Entwicklungspsychologie und Pädagogische Psychologie, 47, 123-138. https://doi.org/10.1026/0049-8637/a000141

Scheerer-Neumann, G. (1989). Rechtschreibschwäche im Kontext der Entwicklung. In I. M. Naegele \& R. Valtin (Hrsg.), LRS in den Klassen 1-10: Handbuch der Lese-Rechtschreib-Schwierigkeiten (S. 25-35). Weinheim: Beltz.

Schneider, W., Stefanek, J. \& Dotzler, H. (1997). Erwerb des Lesens und Rechtschreibens: Ergebnisse aus dem SCHOLASTIK-Projekt. In F. E. Weinert \& A. Helmke (Hrsg.), Entwicklung im Grundschulalter (S. 113-129). Weinheim: Beltz.

Siekmann, K. \& Thomé, G. (2012). Der orthographische Fehler: Grundzüge der orthographischen Fehlerforschung und aktuelle Entwicklungen. Oldenburg: Institut für sprachliche Bildung.

Sparfeldt, J. R., Rost, D. H., Schleebusch, R. \& Heise, A.-L. (2012). Tests und Programme: Lehrerbeurteiltes Schülerverhalten Eine Evaluation der „Lehrereinschätzliste für Sozial- und Lernverhalten" (LSL). Psychologie in Erziehung und Unterricht, 59, $146-158$

Spörer, N., Maaz, K., Vock, M., Schründer-Lenzen, A., Luka, T. \& Bosse, S et al. (2015). Wie entwickeln sich Kinder im inklusiven Unterricht? Zur Anlage der wissenschaftlichen Begleitung des Projekts „Inklusive Grundschule”. In D. Blömer, M. Lichtblau, A.K. Jüttner, K. Koch, M. Krüger \& R. Werning (Hrsg.), Perspektiven auf inclusive Bildung: Gemeinsam anders lehren und lernen (S. 297 - 302). Wiesbaden: Springer VS.

Stevens, J. P. (2002). Applied multivariate statistics for the social science (4th ed.). Hillsdale: Erlbaum.

Stranghöner, D., Hollmann, J., Otterpohl, N., Wild, E., Lütje-Klose, B. \& Schwinger, M. (2017). Inklusion versus Exklusion: Schulsetting und Lese-Rechtschreibentwicklung von Kindern mit Förderschwerpunkt Lernen. Zeitschrift für Pädagogische Psychologie, 31, 125-136. https://doi.org/10.1024/1010-0652/ a000202

Thomé, G. (2003). Entwicklung der basalen Rechtschreibkenntnisse. In U. Bredel, H. Günther, P. Klotz, J. Ossner \& G. SiebertOtt (Hrsg.), Didaktik der deutschen Sprache: Ein Handbuch (Bd. 1, S. 369 - 379). Paderborn: Ferdinand Schöningh.

Valtin, R., Badel, I., Löffler, I., Meyer-Schepers, U. \& Voss, A. (2003). Orthographische Kompetenzen von Schülerinnen und Schülern der vierten Klasse. In W. Bos, E.-M. Lankes, M. Prenzel, K. Schwippert, G. Walther \& R. Valtin (Hrsg.), Erste Ergebnisse aus IG- 
LU: Schülerleistungen am Ende der vierten Jahrgangsstufe im internationalen Vergleich (S. 227 - 264). Münster: Waxmann.

Zöller, I. \& Roos, J. (2009). Einfluss individueller Merkmale und familiärer Faktoren auf den Schriftspracherwerb. In J. Roos \& H. Schöler (Hrsg.), Entwicklung des Schriftspracherwerbs in der Grundschule: Längsschnittanalyse zweier Kohorten über die Grundschulzeit (S. 47-107). Wiesbaden: Verlag für Sozialwissenschaften.

\section{Förderung}

Open Access-Veröffentlichung ermöglicht durch die Universität Potsdam.

\section{Jessica Jaeuthe}

Psychologische Grundschulpädagogik

Institut für Lehrerbildung

Universität Potsdam

Karl-Liebknecht-Str. 24-25

Haus 24

14476 Potsdam

jaeuthe@uni-potsdam.de 